Proceedings

\title{
New Explanation for the Mpemba Effect ${ }^{+}$
}

\section{Ilias J. Tyrovolas}

Greek Ministry of Rural Development \& Foods, Nafplio Regional Quality Control Centre Laboratory, Nafplio 21100, Greece; htyrovolas@minagric.gr

+ Presented at the 5th International Electronic Conference on Entropy and Its Applications, 18-30 November 2019; Available online: https://ecea-5.sciforum.net/.

Published: 17 November 2019

\begin{abstract}
The purpose of this study is to check out the involvement of entropy in Mpemba effect. Several water samples were cooled down to frozen in order to probe if preheat affects the cooling duration time. We found out that preheating of the water sample the cooling duration was reduced. Given this, we theoretically show that water gains more entropy when warmed and re-cooled to the original temperature.
\end{abstract}

Keywords: hydrogen bond network; specific heat; entropy

\section{Introduction}

This case study proposes an explanation for the Mpemba effect, which is considered as the phenomenon wherein, under uncertain conditions, hot water freezes faster than cold water. The fact that the water has been warmed previously contributes to its rapid freezing. Hence many people, when they want to cool water quickly, begin by placing it in the sun. Named after Erasto Mpemba in 1963 [1], the Mpemba effect was reported by Aristotle, Bacon, and Descartes and has been discussed widely in both research as well as popular scientific journals [2]. Auerbach claims that it is different from the supercooling effect [2], but Brownridge argues that it is actually the same [3]. A latest (2016) study [4] disputes the phenomenon at all, although a more recent study [5] shows that the effect is present in granular fluids. Lu and Raz had a generic theory on the Mpemba effect but was not specific applied to water [6]. The explanations have been suggested, can be divided in two general categories. The first one, which for ease can called "physicals", includes theories like these: evaporation [7], frost [8], conduction [9], solutes [10], and supercooling [11]. Second category, called for ease "chemicals", includes theories involving hydrogen bonds such these: crystallization [12], hydrogen bonding [13] and hydrogen bond memory [14]. But, a main query remains unanswered: Why the effect is not always occurs?

In this study, this unique effect is defined as the phenomenon wherein, under certain conditions, it takes a shorter time to cool hot water than to cool cold water: this perspective is adopted by Lu and Raz [6], and described based on macroscopic variables. Further, the underlying mechanism responsible for the effect is proposed and the low repeatability of the phenomenon is explained.

Let us consider two same jars, $\mathrm{A}$ and $\mathrm{B}$, with each containing an equal quantity of water at the same temperature $(T)$, such that the water in A has more entropy than that in $B$. This means that the water molecules of both jars have the same energy; however, those in jar A are moving randomly in all directions, whereas the thermal motion of those in jar B is restricted by the structure mentioned above. Therefore, in the case of the water sample in jar $\mathrm{A}$, random collisions are more likely to occur than in the case of the sample in jar B, resulting in the water molecules losing more kinetic energy (E) on average. This results in a reduction in the temperature according to the relationship $E=(3 / 2) b T$, where $\mathrm{b}$ is the Boltzmann constant. Therefore, the water in jar A cools faster than that in jar B. 
Convection is the dominant form of heat transfer in liquids. According to Newton's law of cooling [15], if we adjust ambient temperature to $\mathrm{Ta}=0 \Rightarrow \Delta \mathrm{T}=\mathrm{T}-\mathrm{Ta}=\mathrm{T}$, during the cooling of a material body, the rate of temperature decrease (cooling rate $=\mathrm{q}$ ) is proportional to the temperature $(T)$ :

$$
q=d T / d t=h T=>T=T_{0} \exp (h t)
$$

where $t$ is the time, $h$ is the heat transfer coefficient, and $T_{0}$ is the initial temperature. The half-time period (HTP) is equal to $\ln 2 / \mathrm{h}$. The greater the value of $h$, the higher is the cooling rate, $q$ so more likely to occur Mpemba effect: this is my aspect discussed below in Discussion section. Heat transfer coefficient (HTC) is dependent upon the physical properties of the water and the physical situation. However, HTC is affected by much many factors such as the container's shape and material and the air circulation within the freezer, among others. For example, Equation (2) applies to a PET bottle assuming a planar geometry [16]:

$$
h=1 /\left(1 / h_{1}+1 / h_{2}+\delta / k\right)
$$

$h$ : overall heat transfer coefficient

$h_{1}$ : heat transfer coefficient inside the bottle

$h_{2}$ : heat transfer coefficient outside the bottle

$\delta$ : PET layer thickness

$k$ : PET thermal conductivity

Thus, the Mpemba effect is hard to predict and is not observed in every instance. We restrict ourselves to the study of the thermodynamics in the single bottle representing a closed thermodynamic system, namely a system that exchanges energy with the environment represented by the cooling chamber. Since we are dealing with phenomena taking place locally and under conditions of local equilibrium inside and outside a typical bottle, all intensive and extensive thermodynamic variables have a space and time dependence [17].

\section{Materials and Methods}

An experiment was performed to elucidate the effect of preheating on the cooling duration, wherein three bottles, A, B, and C, each containing the same quantity of water, were placed in a freezer (Figure A1) with an internal temperature of $-18^{\circ} \mathrm{C}$. The temperature of the water in bottle $\mathrm{C}$ was $50{ }^{\circ} \mathrm{C}$ while that of the water samples in $\mathrm{B}$ and $\mathrm{A}$ was $25^{\circ} \mathrm{C}$. The water in bottle $\mathrm{B}$ was first heated to $50^{\circ} \mathrm{C}$ and then cooled to $25^{\circ} \mathrm{C}$ (Figure 1). After one hour and ten minutes, it was observed that the temperature of the water samples in bottles $B$ and $C$ reached $0{ }^{\circ} \mathrm{C}$ sooner than that for the water in A. It is likely that samples B and C followed the same cooling process; that is to say, the coefficient $h$ was the same for both B and C, while it was larger for A. The HTP for both B and C was the same and lower than that of A. This experiment was conducted at the laboratory of the Nafplio Regional Quality Control Centre, which had met the requirements of the standard ISO/IEC 17025:2005 since 2009 (accreditation body: ESYD S.A., certificate number: 609).

Commercial PET bottles (Figure A2) with a volume of $500 \mathrm{~mL}$ were filled with $500.00 \mathrm{~g}$ of degased (Figure A3) distilled water produced by a distiller (Bibby Sterilin, model: A 4000D, conductivity of water: $1-2 \mu \mathrm{S} / \mathrm{cm}$ ). The ambient temperature within the laboratory was adjusted to $25^{\circ} \mathrm{C}$. The water in bottle B was heated to $50{ }^{\circ} \mathrm{C}$ in a Pyrex glass using a hot plate (Figure A4) and then left to cool to $25{ }^{\circ} \mathrm{C}$. The water in bottle $\mathrm{C}$ was heated to $50{ }^{\circ} \mathrm{C}$. The water was poured immediately into the bottles, which were at the ambient temperature. The sensors (pins) of the digital alert thermometers used for the temperature measurements were inserted from the top to the middle of the bottles. With no relaxation time, the bottles were capped then immediately placed in a freezer and cooled to $0^{\circ} \mathrm{C}$ (Figure 2). 


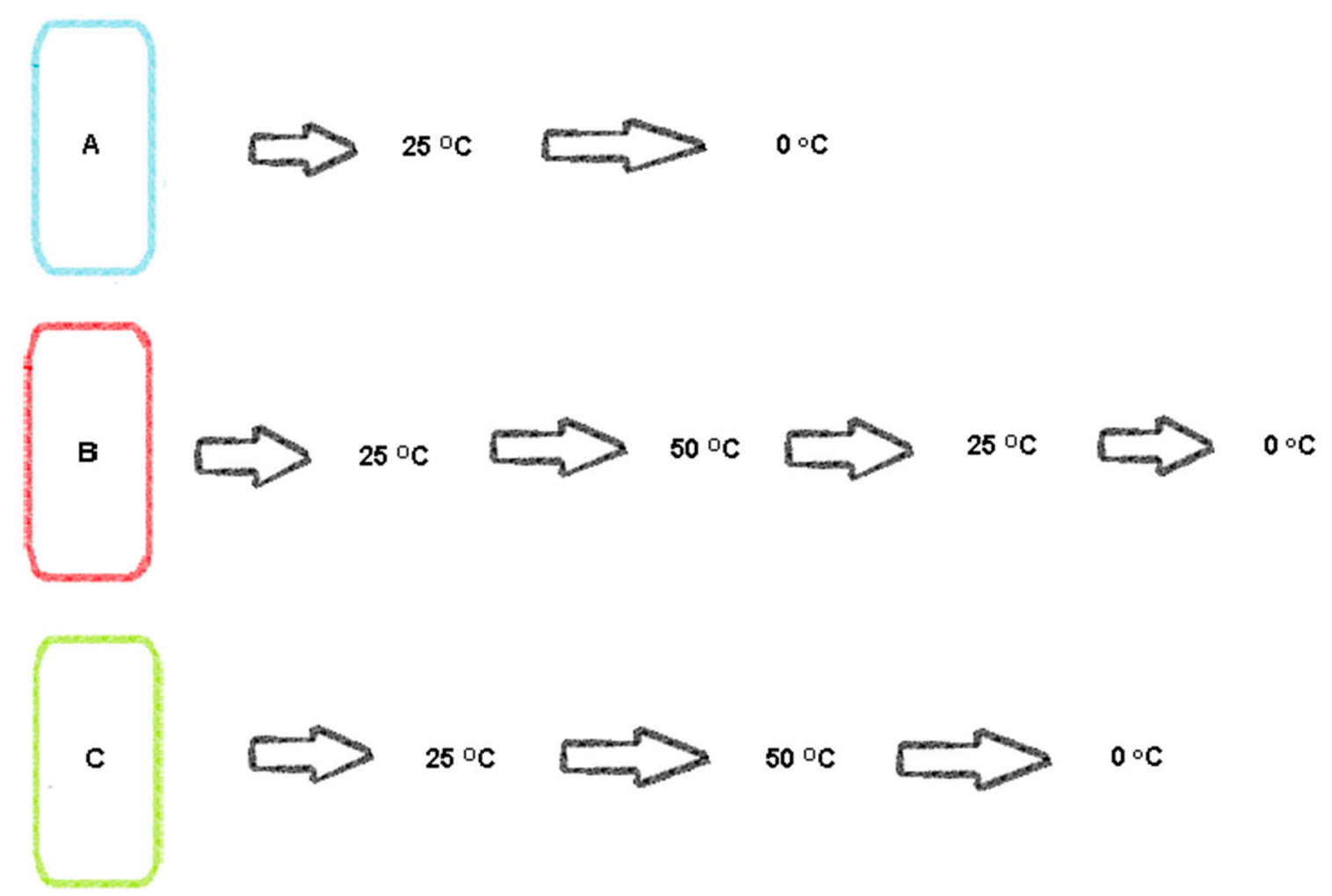

Figure 1. Experimental plan

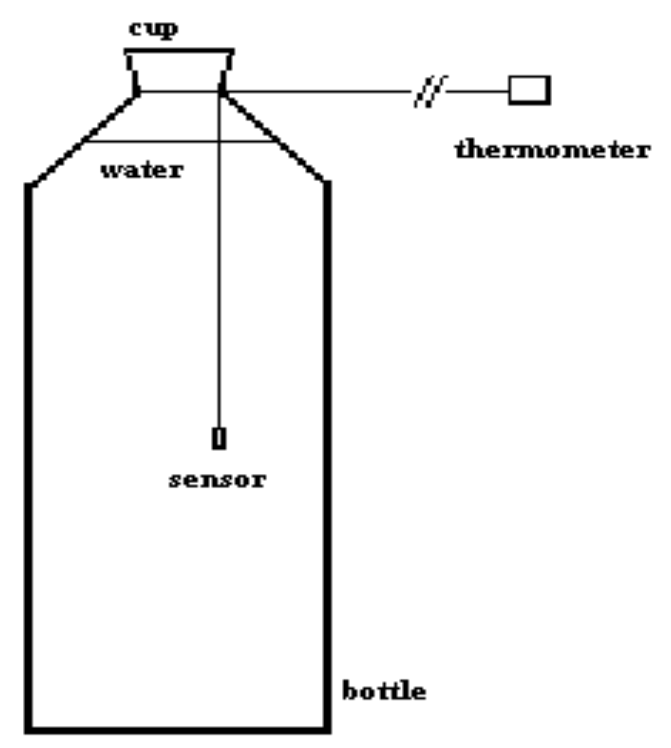

Figure 2. Experimental layout.

Digital alert timers were used to measure the duration time. Measurements were carried out under the plan one bottle per day. Initial conditions are kept the same in the different repeated runs.

\section{Results}

The experimental results are shown in Table 1 arranged in descending order: 
Table 1. Cooling duration.

\begin{tabular}{cccccccccccccc}
\hline Process & \multicolumn{1}{c}{ Time (min) } & \multicolumn{10}{c}{ Average } & S.D. \\
\hline A & 101 & 100 & 100 & 100 & 100 & 100 & 100 & 100 & 100 & 99 & 100 & 0.5 \\
B & 99 & 99 & 98 & 98 & 97 & 97 & 97 & 97 & 96 & 96 & 97.4 & 1.1 \\
C & 98 & 98 & 97 & 97 & 97 & 97 & 97 & 97 & 96 & 96 & 97 & 0.7 \\
\hline
\end{tabular}

Data are illustrated in Figure 3. The average values were compared using the F test. First, the average value of the A samples (Aav) was compared with those of samples B and C ([Bav + Cav]/2), in order to check whether preheating affected the duration of cooling. Next, Bav and Cav were compared to determine whether they were equal within the limits of experimental uncertainty. In the first case, the F value, F1, was calculated to be 86.0. Further, the F tables with a significance level of $0.1 \%$ gave $\mathrm{F}=13.6<\mathrm{F} 1$, showing that the preheating of the water strongly affected the cooling duration. In the second case, F2 was calculated to be 1.3, which is significantly less than 13.6 (F1). This meant that Bav $=$ Cav.

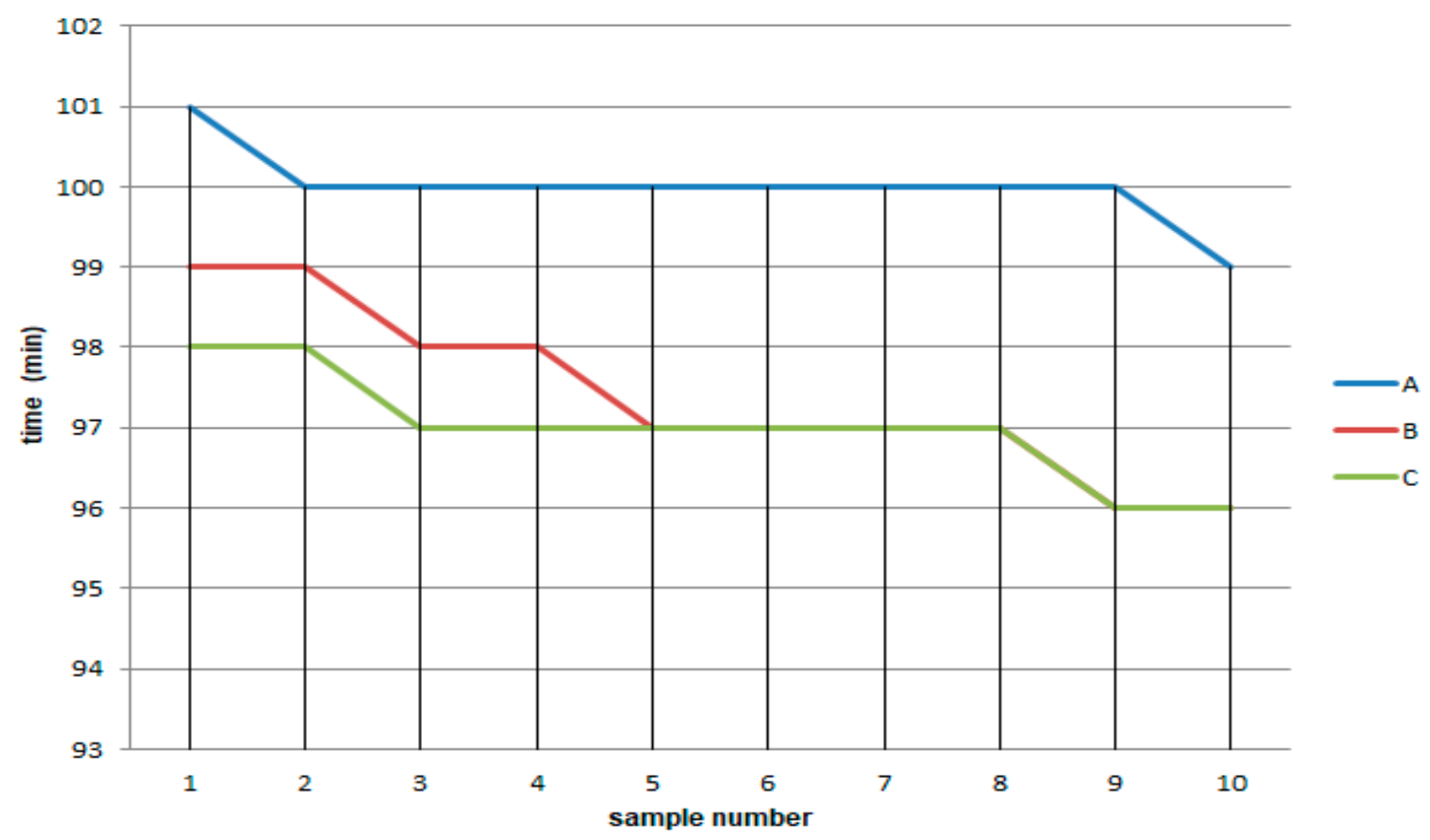

Figure 3. The three processes.

\section{Discussion}

Water molecules are V-shaped electric dipoles. Cold water has a rudimentary structure, hereinafter referred to as simply the "structure." The structure consists of intermolecular cyclic associates (clusters) of water with the general formula $\left(\mathrm{H}_{2} \mathrm{O}\right) \mathrm{n}$ [18]. The ordering of water molecules into associates corresponds to a decrease in their entropy (randomness) [18]. Each water molecule can form two hydrogen bonds $(\mathrm{O}-\mathrm{H})$ involving their hydrogen atoms plus two further hydrogen bonds $(\mathrm{O}: \mathrm{H})$ utilizing the hydrogen atoms attached to neighboring water molecules. These four hydrogen bonds optimally arrange themselves in a tetrahedral structure around each water molecule, as observed in ordinary ice [19]. Heating raises water energy by $\mathrm{H}-\mathrm{O}$ thermal contraction and $\mathrm{O}: \mathrm{H}$ expansion [20], which can be deemed as a raise in entropy. In liquid water, thermal energy bends, stretches, exchanges and sometimes breaks these hydrogen bonds. However, the average structure of a water molecule is similar to this tetrahedral arrangement [19]. Today, this tetrahedrally coordinated water structure is generally accepted; however, the arrangement of most hydrogen-bonded molecules is not symmetrical. At room temperature, about $80 \%$ of the molecules of liquid water have one strongly hydrogen-bonded O-H group and one non-or only weakly bonded 
$\mathrm{O}-\mathrm{H}$ group at any instant. The remaining $20 \%$ of the molecules are made up of four-hydrogen-bonded tetrahedrally coordinated clusters [19]. The average energy of the hydrogen bonds between the $\mathrm{H}_{2} \mathrm{O}$ molecules during the process of cluster formation is $0.1067 \pm 0.0011 \mathrm{eV}$. As the energy of the hydrogen bonds between the $\mathrm{H}_{2} \mathrm{O}$ molecules increases to $0.14 \mathrm{eV}$, the water clusters are destroyed [18]. A typical cluster consists of five water molecules. In ice, this tetrahedral clustering is extensive, producing crystalline structures [19]. In liquid water, the tetrahedral clustering is found locally and reduces with increasing temperature [19]. These local structures contribute to the anomalies of water [21]. Water primarily consists of low-density clusters of mostly tetrahedrally hydrogen-bonded water molecules and high-density clusters with many long, weak, and bent hydrogen bonds plus many close, non-hydrogen-bonded, van der Waals interactions [19]. This spatial organization is known as low-density-liquid (LDL) like and high-density -liquid (HDL) like environments [21]. Such liquid water behaves as though it is a mixture of two liquids that change relative composition with variations in temperature and pressure [19]. When decreasing the temperature, the liquid undergoes a structural transformation coinciding with the onset of an extended hydrogen bond network [22]. In bulk water, at any instant, it is expected that strongly tetrahedrally oriented hydrogen bonds form a network (lattice), with a small number of isolated pockets of water molecules with weak or broken hydrogen bonds also being present [19]. Most of the interesting properties of water come from this three-dimensional hydrogen-bonding network [22]. The hydrogen bond energy is $5-10 \mathrm{kcal} / \mathrm{mole}$, while the energy of $\mathrm{O}-\mathrm{H}$ covalent bond in the $\mathrm{H}_{2} \mathrm{O}$ molecule is $109 \mathrm{kcal} / \mathrm{mole}$. The average energy $(\Delta \mathrm{E} \mathrm{H} \ldots \mathrm{O})$ of the hydrogen $\mathrm{H} \ldots \mathrm{O}$ bonds between $\mathrm{H}_{2} \mathrm{O}$ molecules is $0.1067 \pm 0.0011 \mathrm{eV}$. With fluctuations in the temperature of water, the average energy of the hydrogen H...O bonds in the water molecule clusters changes [18]. This is the reason that the hydrogen bonds in the liquid state are relatively weak and unstable: it is thought that they form and break readily with changes in the temperature. It is known that thermal oscillations (fluctuations) lead to the bending and breaking of hydrogen bonds [18,19]. When water is heated, the hydrogen bonds break, and the molecules move further apart and get repositioned randomly, resulting in extensive collapse of the structure. Hence, the fraction of water molecules joined by hydrogen bonds decreases. According to theoretical calculations, heating to $40{ }^{\circ} \mathrm{C}$ breaks approximately half the hydrogen bonds in water associates [18]. The breaking of these bonds and the resulting increase in the degree of disorder of the water molecules leads to increased entropy (S). The increase in the internal entropy (dS) when water is heated from a lower temperature $T_{1}$ to a higher temperature $T_{2}$ can be calculated as follows [17]:

$$
d S_{1}{ }^{2}=\operatorname{MCln}\left(T_{2} / T_{1}\right)
$$

where $M$ is the mass of water and $C$ is the specific heat. My aspect is that the lattice does not extensively re-form immediately upon cooling, as the reconstruction process requires time. If the cooling process is very fast and performed using a freezer, the water molecules do not get sufficient time to restructure. In contrast, when water remains for a long time in a fridge, wherein a stable temperature of $5{ }^{\circ} \mathrm{C}$ is maintained, the water molecules have sufficient time to reorder. Entropy is the measure of the disorder. When the water is cooled to an initial low temperature, the structure does not form instantaneously, that is, its internal entropy does not decrease immediately: the disordering process requires some time to totally reversed. Supposed $T_{1}<T_{2}$, only for a reversed process or cycle can be $d S_{1}{ }^{2}=d S_{2}{ }^{1}$, while for an unreversed process it is always $d S_{1}{ }^{2}>d S_{2}{ }^{1}$ [17]: during the cooling process, the water structure does not instantaneously return to the ordered state, as hydrogen-bond network does not rectify instantly in its whole extension.

Considering a cycle $T_{1} \rightarrow T_{2} \rightarrow T_{1}$ (Figure 4 ) (in our experiment $T_{1}=25^{\circ} \mathrm{C}, T_{2}=50^{\circ} \mathrm{C}$ ) it is:

$$
d S_{1}{ }^{2} \geq d S_{2}{ }^{1} \text { or } d S h \text { (heating) } \geq d S c \text { (cooling) }
$$

- If $\mathrm{d} \mathrm{S}_{\mathrm{h}}=\mathrm{d} \mathrm{S}_{\mathrm{c}}$ the Mpemba effect does not happen (slow process - low HTC)

- If $\mathrm{dS}_{\mathrm{h}}>\mathrm{dS}$ c the Mpemba effect occurs (fast process - high HTC) 
Entropy for a defined body is a state function and i.e., for $T_{1}$ is given by integration of (3) thus: $\mathrm{S}_{1}$ $=\mathrm{MClnT} 1$, but here they are two different water bodies with different "structures": one before heating and the other after re-cooling. The different "structure" implies different specific heats. The last inequality above combined with (3) results to inequality of the specific heats:

$$
d S h>d S c \Leftrightarrow M C h \ln \left(T_{2} / T_{1}\right)>M C c \ln \left(T_{1} / T_{2}\right)=-M C c \ln \left(T_{2} / T_{1}\right)=>C c>C h
$$

Specific heat is not a constant but varies with temperature [17] which affects the network's extent $[19,22]$. Specific heat of water is in average $1 \mathrm{cal} / \mathrm{gr}{ }^{\circ} \mathrm{C}=4.19 \mathrm{~J} / \mathrm{gr}{ }^{\circ} \mathrm{C}$ in the range $25^{\circ} \mathrm{C}-60{ }^{\circ} \mathrm{C}$ [23]. Anyhow specific heat is affected by the network's extent [19]. This thermodynamic process or cycle can be visualized in a "temperature vs entropy diagram" or T-S diagram (Figure 4) [24]. The curve for entropy reduction as a function of the temperature, $S_{c}=f(T)$, lags relative to the entropy growth curve $S_{h}=f(T)$ :

$$
C h<C c=>M C h l n T<M C c l n T \Leftrightarrow S h<S c
$$

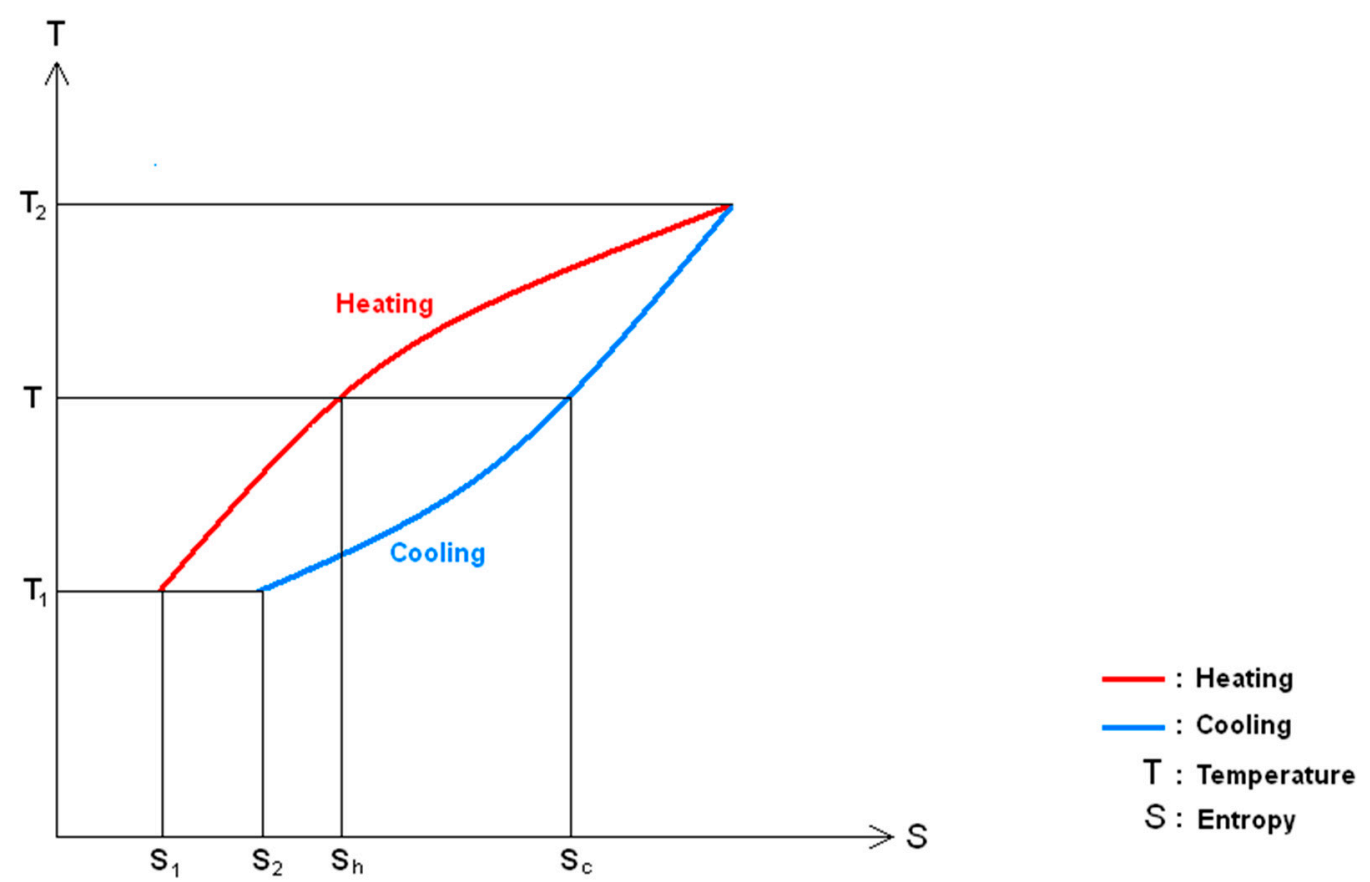

Figure 4. Curves of entropy as function of temperature.

As we see in Figure 4, at any temperature $T$, the entropy during heating, $S_{h}$, is less than the entropy during cooling, $S_{c}$. After being heated and then cooled to the starting temperature, the water now has greater entropy and fewer hydrogen bonds than it did immediately prior to being heated, even though the temperature is now the same i.e., for $T_{1}$ it is $S_{1}<S_{2}$. Indeed, water behaves differently, and possesses a different structuring, at the same temperature depending upon whether it is being heated or cooled [25].

\section{Conclusions}

Warm water is cooling faster than cold because it contains more entropy when it comes to its temperature. The lattice structure is more extended in cold water. Any dissolved salts present in the water change the structure of the water molecules [26] because ions are hydrated [27]. Consequently, in the case of water containing dissolved ions, the water network is smaller, and the molecules are less organized than in pure water. Therefore, the effect of preheating is expected to be weaker. 


\section{Appendix A}

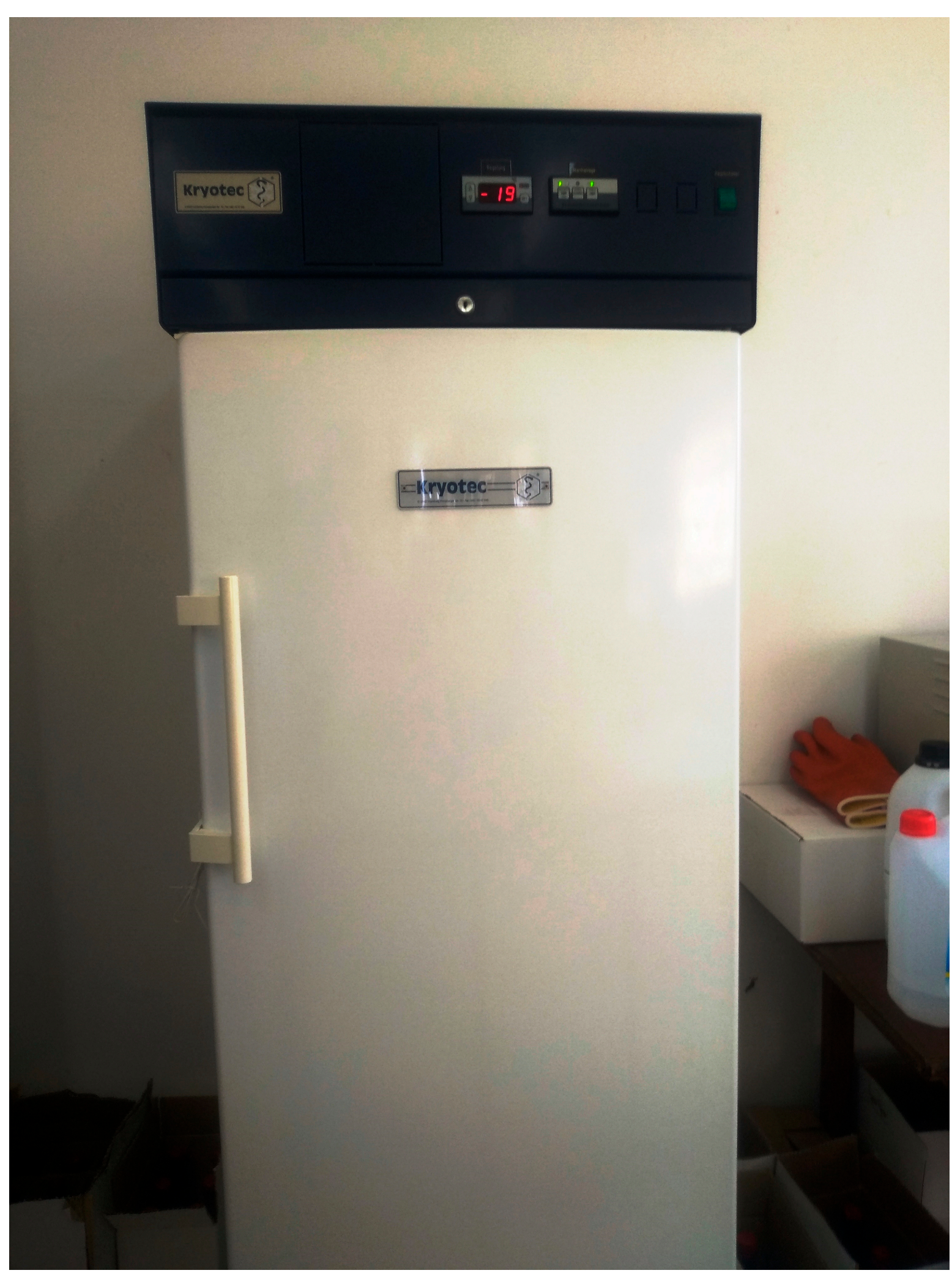

Figure A1. Freezer. 


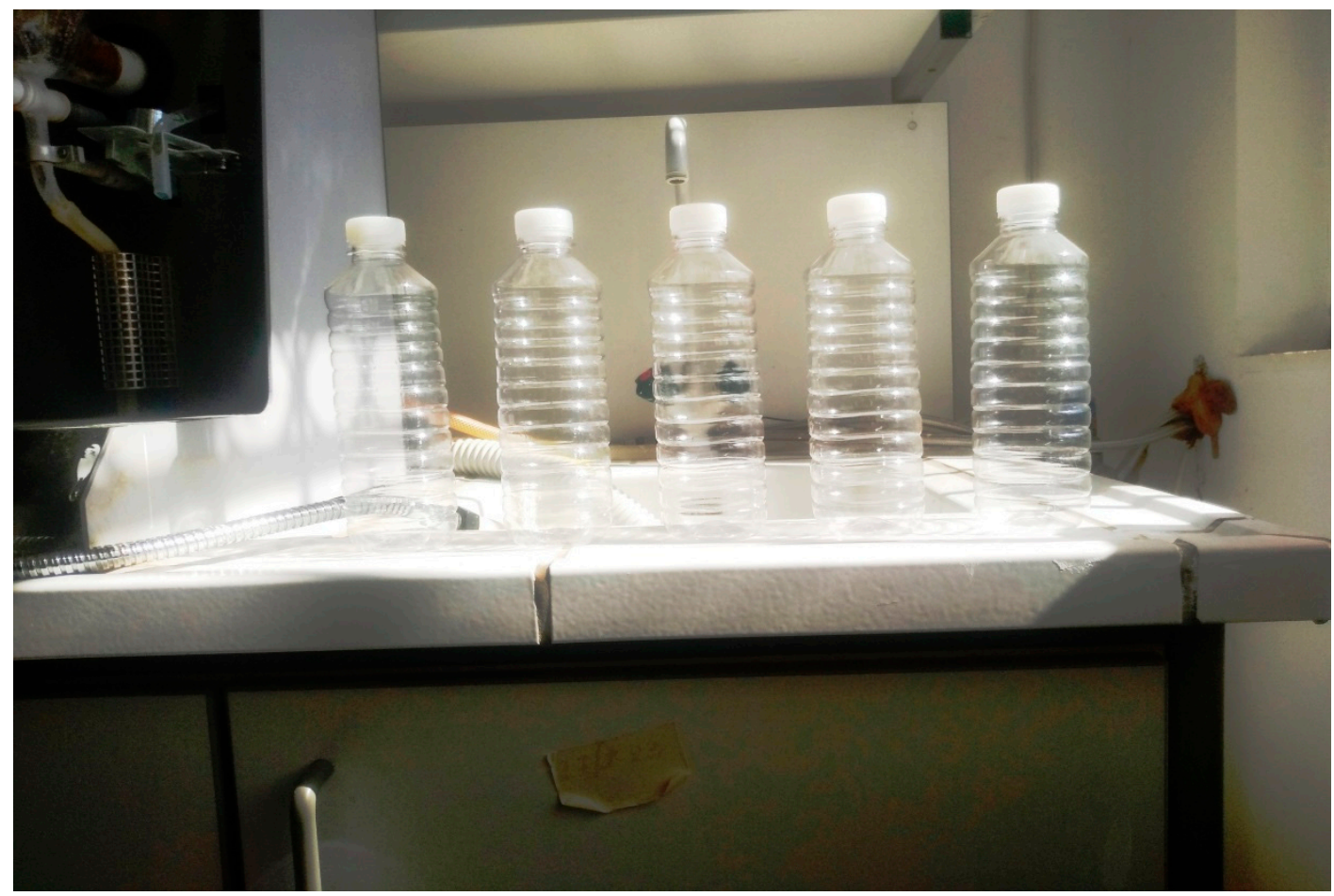

Figure A2. PET bottles.

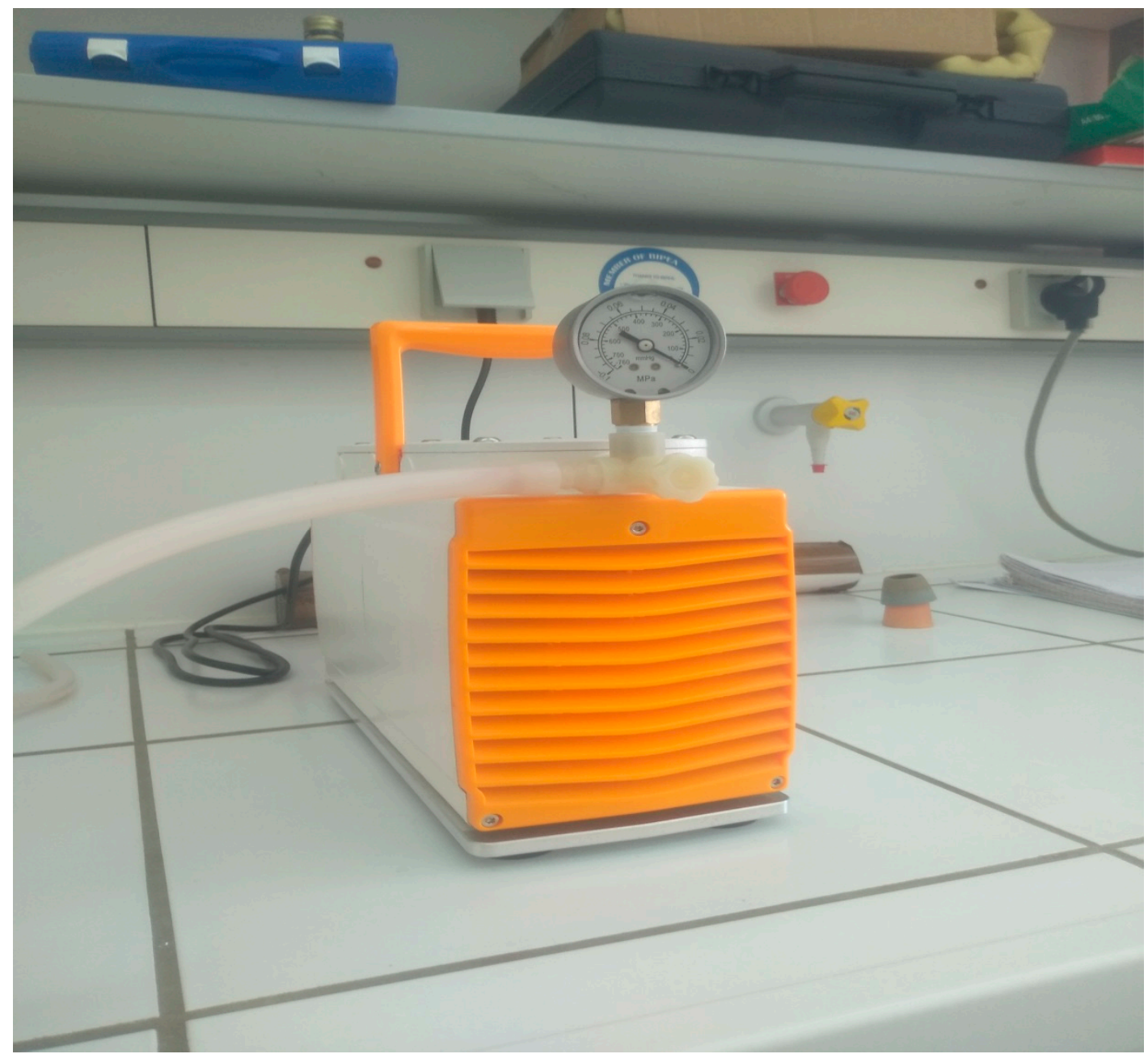

Figure A3. Degaser. 


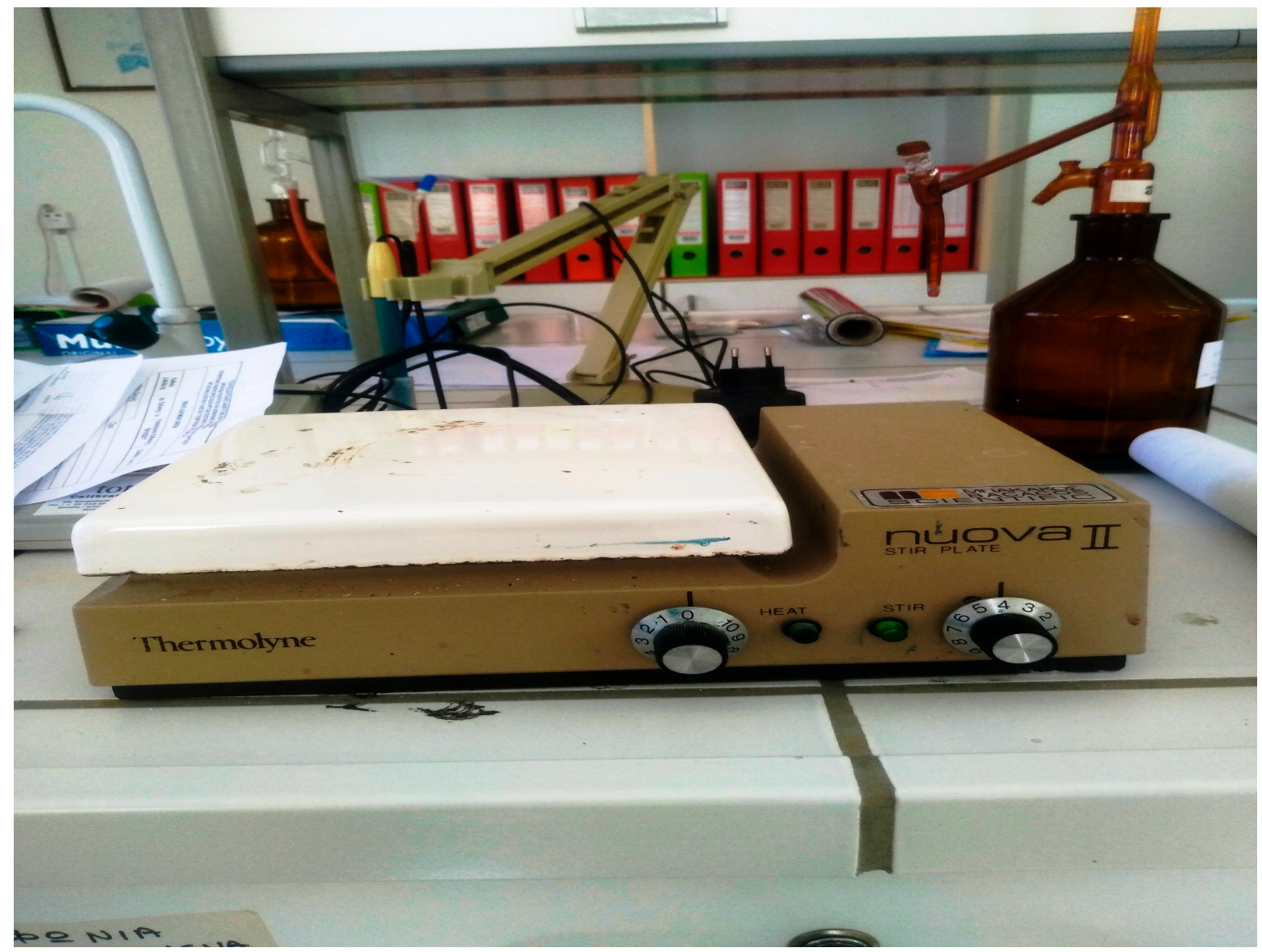

Figure A4. Heat plate.

\section{References}

1. Mpemba, E.; Osborne, D. Cool? Phys. Educ. 1979, 14, 410. doi:10.1088/0031-9120/14/7/312

2. Auerbach, D. Supercooling and the Mpemba effect: When hot water freezes quicker than cold. Am. J. Phys. 1995, 63, 882. doi:10.1119/1.18059

3. Brownridge, J. Physics. A search for the Mpemba effect: When hot water freezes faster than cold. physics. pop-ph 2010. Available online: https://arxiv.org/abs/1003.3185

4. Burridge, H.C.; Linden, P.F. Questioning the Mpemba effect: Hot water does not cool more quickly than cold. Sci. Rep. 2016, 6, 37665. doi:10.1038/srep37665

5. Lasanta, A.; Vega Reyes, F.; Prados, A.; Santos, A. When the hotter cools more quickly: Mpemba effect in granular fluids. Phys. Rev. Lett. 2017, 119, 148001, doi:10.1103/PhysRevLett.119.148001

6. Lu, Z.; Raz, O. Nonequilibrium thermodynamics of the Markovian Mpemba effect and its inverse. Proc. Natl. Acad. Sci. USA 2017, 114, 5083-5088. doi:10.1073/pnas.1701264114

7. Kell, G.S. The freezing of hot and cold water Am. J. Phys. 1969, 37, 564-565. doi:10.1119/1.1975687

8. Jeng, M. Hot water can freeze faster than cold? Am. J. Phys. 2006, 74, 514. doi:10.1119/1.2186331

9. Kowalewski, T. Experimental Methods for Quantitative analysis of thermally driven flows, Phase Change with Convection, Modeling and Validation. In International Centre for Mechanical Sciences (Courses and Lectures); Kowalewski, T.A., Gobin, D., Eds.; Springer-Verlag Wien: New York, NY, USA, 2004; Volume 449, pp. 171-218.

10. Katz, J.I. When hot water freezes before cold. Am. J. Phys. 2009, 77, 27-29. doi:10.1119/1.2996187

11. Vynnycky, M.; Kimura, S. Can natural convection alone explain the Mpemba effect? Int. J. Heat Mass Transf. 2015, 80, 243-255, doi:10.1016/j.ijheatmasstransfer.2014.09.015

12. Jin, J.; Goddard, W.A. Mechanisms Underlying the Mpemba Effect in Water from Molecular Dynamics Simulations. J. Phys. Chem. C 2015, 119, 2622-2629. doi:10.1021/jp511752n 
13. Tao, Y.; Zou, W.; Jia, J.; Li, W.; Cremer, D. Different Ways of Hydrogen Bonding in Water-Why Does Warm Water Freeze Faster than Cold Water? J. Chem. Theory Comput. 2017, 13, 55. doi:10.1021/acs.jctc.6b00735

14. Zhang, X.; Huang, Y.; Ma, Z.; Zhou, Y.; Zhou, J.; Zheng, W.; Jiang, Q.; Sun, C.Q. Hydrogen-bond memory and water-skin supersolidity resolving the Mpemba paradox. Phys. Chem. Chem. Phys. 2014, 16, 2299523002. doi:10.1039/C4CP03669G

15. O' Sullivan, C. Newton's law of cooling-A critical assessment Am. J. Phys. 1990, 58, 956-960. doi:10.1119/1.16309

16. Liga, A.; Montesanto, S.; Mannella, G.; La Carrubba, V.; Brucato, V.; Cammalleri, M. Study on heat transfer coefficients during cooling of PET bottles for food beverages. Heat Mass Transf. 2016, 52, 14791488. doi:10.1007/s00231-015-1652-x

17. Kondepudi, D.; Prigogine, I. Modern Thermodynamics: From Heat Engines to Dissipative Structures; Wiley: Chichester, UK, 2002; pp. 54-82.

18. Ignatov, I.; Mosin, O.V. Structural Mathematical Models Describing Water Clusters. Nanotechnol. Res. Pract. 2014, 3, 141. doi:10.13187/ejnr.2014.3.141

19. Chaplin, M.F. Structure and Properties of Water in its Various States. In: Encyclopedia of Water, P.Maurice, Wiley, 2019; pp.1-19. doi:10.1002/9781119300762.wsts0002

20. Huang, Y.; Zhang, X.; Ma, Z.; Zhou, Y.; Zheng, W.; Zhu, J.; Sun, C. Hydrogen-bond relaxation dynamics: Resolving mysteries of water ice. Coord. Chem. Rev. 2015, 285, 109-165. doi:10.1016/j.ccr.2014.10.003

21. Martelli, F. Unravelling the contribution of local structures to the anomalies of water: The synergistic action of several factors. J. Chem. Phys. 2019, 150, doi:10.1063/1.5087471

22. Mallamace, F.; Corsaroa, C.; Stanley, H. Possible relation of water structural relaxation to water anomalies Proc. Natl. Acad. Sci. USA 2013, 110, 4899-4904. doi:10.1073/pnas.1221805110

23. Pramuditya, S. Water Thermodynamic Properties. ITB Physics Department-Technical Document. Available online: https://syeilendrapramuditya.wordpress.com/2011/08/20/water-thermodynamic-properties

24. Temperature Entropy Diagram-Thermodynamics. Engineers Edge. http://www.engineersedge.com/thermodynamics/temp_enthalpy_th_diagram.htm

25. Pang, X.; Deng, B. Infrared absorption spectra of pure and magnetized water at elevated temperatures. Europhys. Lett. 2010, 92, 65001. doi:10.1209/0295-5075/92/65001

26. Hribar, B.; Southall, N.; Vlachy, V.; Dill, K. How Ions Affect the Structure of Water. J. Am. Chem. Soc. 2002, 124, 12302-12311, doi:10.1021/ja026014h

27. Ahmed, M.; Namboodiri, V.; Singh, A.; Mondal, J.; Sarkar, S. How Ions Affect the Structure of Water: A Combined Raman Spectroscopy and Multivariate Curve Resolution Study. J. Phys. Chem. B 2013, 117, 16479-16485. doi:10.1021/jp4100697

(C) 2019 by the authors. Licensee MDPI, Basel, Switzerland. This article is an open access article distributed under the terms and conditions of the Creative Commons Attribution (CC BY) license (http://creativecommons.org/licenses/by/4.0/). 